\title{
Cardiovascular prevention starts from your mouth
}

Stefano Masi ${ }^{1,2}$, Francesco D’Aiuto $^{3}$, John Deanfield ${ }^{1}$

1. National Centre for Cardiovascular Prevention and Outcomes, Institute of Cardiovascular Science, University College London, UK

2. Department of Clinical and Experimental Medicine, University of Pisa, Italy

3. UCL Eastman Dental Institute, Periodontology Unit, University College London, UK

Editorial to the manuscript entitled: "Improved Oral Hygiene Care Attenuates the Cardiovascular Risk of Oral Health Disease: A Population-based Study from Korea” by Kang et al.

Total number of words: 1408

\section{Corresponding author:}

Stefano Masi, MD, PhD, FESC

National Centre for Cardiovascular Prevention and Outcomes

Institute of Cardiovascular Science

University College London

Level 2, Nomura House

1 St Martins Le Grand

London EC1A 4NP

UK

$\&$

Department of Clinical and Experimental Medicine

Università di Pisa

Via Roma 67

56126 Pisa

Italy 
Atherosclerosis remains the leading cause of cardiovascular disease worldwide and recent clinical evidence has confirmed that inflammation plays a significant role in its complications ${ }^{1}$. Diseases characterised by a chronic inflammatory exposure (including rheumatoid arthritis, psoriasis, oral inflammatory diseases, chronic bowel diseases, etc.) have been associated with raised risk of future cardiovascular events and have been studied to explore the potential impact of anti-inflammatory drugs or procedures on mitigating the cardiovascular risk ${ }^{2}$. The ability to modulate inflammation in these conditions has also enabled identification of inflammatory pathways potentially involved in the regulation of vascular homeostasis. The association between parameters of poor oral health and risk of cardiovascular diseases has been repeatedly reported by observational (cross-sectional, case controls and prospective) studies in humans, leading to the inclusion of periodontitis (a common oral inflammatory disease) in the group of potential conditions increasing the risk of cardiovascular disease in the most recent European Guidelines for cardiovascular disease prevention ${ }^{3}$. The interest around the potential link between oral health and cardiovascular disease is explained by its potential benefits in term of cardiovascular disease prevention. Indeed, oral diseases are highly prevalent in the general population, collectively affecting 3.9 billion people worldwide ${ }^{4}$. This prevalence is significantly higher than other common cardiovascular risk factors (i.e. diabetes) and if a causal link with atherosclerosis could be demonstrated, the proportion of individuals in the general population who might obtain cardiovascular benefits from an improved oral health could be substantial, with a significant reduction of the costs for the public health systems.

In this issue of the European Heart Journal, Kang et al. report the results of a large, prospective, population-based study exploring the potential influence of oral health care on the future risk of cardiovascular disease ${ }^{5}$. Using data from the National Health Insurance System-National Health Screening Cohort (NHISHEALS), including almost the entire population in Korea, the authors documented that an increased number of dental caries, the presence of periodontitis and a greater loss of teeth were associated with an increased risk of future major cardiovascular events (MACEs), including cardiovascular death, acute myocardial infarction, heart failure, and stroke. Importantly, frequent tooth brushing and regular dental visits were associated with a lower cardiovascular disease risk and mitigated the risk of cardiovascular events in participants with oral diseases. While, the association between periodontitis and adverse cardiovascular events lost statistical significance after multivariable adjustments, including age, hypertension, diabetes, and tobacco smoking, measures of dental caries and tooth loss remained significantly associated with the risk of MACEs in the fully adjusted model. Based on these results, the authors concluded that oral health promotion strategies (i.e. better dental hygiene behaviour) could modify the cardiovascular risk associated with poor oral health. 
The results by Kang et al. confirms previous studies suggesting a potential impact of oral inflammatory diseases on the risk of cardiovascular events, over and above the contribution of traditional cardiovascular risk factors ${ }^{6}$. They also provide clinical evidence for a potential benefit in cardiovascular protection achieved by the simple adoption of better oral health behaviours in a large prospective study. This is important as current guidelines recognise the general lack of knowledge on the potential cardiovascular benefits obtained with active treatment of oral health diseases ${ }^{3}$.

The biological pathways accounting for the results presented by Kang et al. remain to be clarified. We have previously documented that periodontitis and an intensive treatment regimen able to improve gingival health can alter systemic inflammation and have profound effects on endothelial cell function and biology ${ }^{7}$. More recently, we confirmed that the same treatment improves metabolic control, endothelial function and prevent renal deterioration in patients with type 2 diabetes ${ }^{8}$. Based on these results and considering the elevated systemic inflammatory burden commonly observed in patients with diabetes and oral diseases, it could be speculated that the systemic benefits obtained with an improved oral health could depend on a reduced exposure to chronic systemic inflammation (Figure 1). As highlighted by Kang et al., this hypothesis is in keeping with the results of the CANTOS trail, showing that reduction of systemic inflammation reduces the risk of cardiovascular events and mortality in patients with established atherosclerosis ${ }^{9}$. However, while in patients with diabetes we were able to document a significant reduction of C-reactive protein after intensive periodontal treatment compared to the control group, we could not find any associations between changes of this and other inflammatory markers and the improved endothelial function ${ }^{7,8}$. The results by Kang et al. somewhat confirm that, over and above inflammation, there might be other factors accounting for the link between oral diseases and cardiovascular health. Indeed, dental caries are commonly associated with a lower systemic inflammatory exposure compared to periodontal diseases and, despite this, they had a stronger association with adverse cardiovascular events. The systemic inflammatory response in patients suffering from oral diseases is thought to depend on the translocation of pathogenic bacteria from the oral cavity to the systemic circulation ${ }^{6}$. Significant interest in recent years has focused on the microbiota-host interactions, as accumulating evidence has revealed that microbiota might play an important role in cardiovascular diseases. Changes in the composition of oral microbiota, referred to as dysbiosis, have been suggested as potential causes of oral disease ${ }^{10}$ and oral bacteria have been isolated from atherosclerotic plaques ${ }^{11}$ (Figure 1). Thus, a dysbiosis that promotes the evolution of oral disease might also contribute to the development of atherosclerosis with mechanisms that are not reflected necessarily by the levels of C-reactive protein. The role of oral bacteria as central mediators of the link between oral and cardiovascular diseases might also account for the benefits of frequent tooth brushing and regular dental visits described by Kang et al. Indeed, these procedures are associated with better control of the microbial plaque. 
The relationship between oral health and cardiovascular disease might also lie in the increase of systemic oxidative stress (Figure 1). This might affect the risk of cardiovascular events and progression of the oral disease by altering the function of immune-inflammatory cells by two separate mechanisms. Firstly, while intracellular oxidative stress might reduce NO availability thus promoting endothelial dysfunction, it might also modify the pattern of cytokines produced by immuneinflammatory cells, reducing their capacity to contrast the bacterial aggression to the oral cavity ${ }^{12}$. We recently reported that production of mitochondrial reactive oxygen species in immuneinflammatory cells is influenced by periodontal treatment, represents the strongest predictor of the changes in endothelial function and is accompanied by changes in the production of cytokines with an essential role in the regulation of the innate as well as adaptive immunity ${ }^{13}$. Secondly, chronic activation of the immune-inflammatory cells and exposure to elevated levels of oxidative stress might lead to their exhaustion, ultimately resulting in cellular ageing (Figure 1). We have documented that patients with chronic periodontitis have shorter leukocyte telomere length (LTL) and that the size of telomeres is directly related to circulating levels of oxidative stress and the severity of the oral diseases $^{14,15}$. In turn, telomere shortening has been associated with cardiovascular disease ${ }^{16}$, and cells with critically short telomeres might favour progression of the oral disease, as they produce higher levels of proinflammatory cytokines and lose their capacity to respond effectively to bacterial aggressions.

In summary, the study by Kang et al. has the merit of confirming the strong link between oral health and the risk of cardiovascular diseases. For the first time, it also provides evidence of the potential impact of better dental hygiene on the future risk of cardiovascular events/mortality. While large clinical trials assessing the impact of better oral health on hard cardiovascular outcomes are still lacking and the mechanisms accounting for the potential reduction of cardiovascular disease risk achieved with better oral health remain largely unknown, this manuscript adds further on the role of poor oral health onto cardiovascular diseases. Given that periodontal diseases are the commonest form of inflammatory/infectious diseases worldwide, the public health benefits of promoting oral health are potentially large. 


\section{REFERENCES}

1. Libby P, Loscalzo J, Ridker PM, Farkouh ME, Hsue PY, Fuster V, Hasan AA, Amar S. Inflammation, Immunity, and Infection in Atherothrombosis: JACC Review Topic of the Week. Journal of the American College of Cardiology 2018;72(17):2071-2081.

2. Tabas I, Glass CK. Anti-inflammatory therapy in chronic disease: challenges and opportunities. Science 2013;339(6116):166-72.

3. Piepoli MF, Hoes AW, Agewall S, Albus C, Brotons C, Catapano AL, Cooney MT, Corra U, Cosyns B, Deaton C, Graham I, Hall MS, Hobbs FDR, Lochen ML, Lollgen H, Marques-Vidal P, Perk J, Prescott E, Redon J, Richter DJ, Sattar N, Smulders Y, Tiberi M, van der Worp HB, van Dis I, Verschuren WMM, Binno S, Group ESCSD. 2016 European Guidelines on cardiovascular disease prevention in clinical practice: The Sixth Joint Task Force of the European Society of Cardiology and Other Societies on Cardiovascular Disease Prevention in Clinical Practice (constituted by representatives of 10 societies and by invited experts)Developed with the special contribution of the European Association for Cardiovascular Prevention \& Rehabilitation (EACPR). European heart journal 2016;37(29):2315-2381.

4. Marcenes W, Kassebaum NJ, Bernabe E, Flaxman A, Naghavi M, Lopez A, Murray CJ. Global burden of oral conditions in 1990-2010: a systematic analysis. Journal of dental research 2013;92(7):592-7.

5. Park SY, Kim SH, Kang SH, Yoon CH, Lee HJ, Yun PY, Youn TJ, Chae IH. Improved oral hygiene care attenuates the cardiovascular risk of oral health disease: a population-based study from Korea. European heart journal 2019;40(14):1138-1145.

6. Tonetti MS, Van Dyke TE, working group 1 of the joint EFPAAPw. Periodontitis and atherosclerotic cardiovascular disease: consensus report of the Joint EFP/AAP Workshop on Periodontitis and Systemic Diseases. Journal of periodontology 2013;84(4 Suppl):S24-9.

7. Tonetti MS, D'Aiuto F, Nibali L, Donald A, Storry C, Parkar M, Suvan J, Hingorani AD, Vallance P, Deanfield J. Treatment of periodontitis and endothelial function. The New England journal of medicine 2007;356(9):911-20.

8. D'Aiuto F, Gkranias N, Bhowruth D, Khan T, Orlandi M, Suvan J, Masi S, Tsakos G, Hurel S, Hingorani AD, Donos N, Deanfield JE, Group T. Systemic effects of periodontitis treatment in patients with type 2 diabetes: a 12 month, single-centre, investigator-masked, randomised trial. The lancet Diabetes \& endocrinology 2018;6(12):954-965.

9. Ridker PM, Everett BM, Thuren T, MacFadyen JG, Chang WH, Ballantyne C, Fonseca F, Nicolau J, Koenig W, Anker SD, Kastelein JJP, Cornel JH, Pais P, Pella D, Genest J, Cifkova R, Lorenzatti A, Forster T, Kobalava Z, Vida-Simiti L, Flather M, Shimokawa H, Ogawa H, Dellborg M, Rossi PRF, Troquay RPT, Libby P, Glynn RJ, Group CT. Antiinflammatory Therapy with Canakinumab for Atherosclerotic Disease. The New England journal of medicine 2017;377(12):1119-1131.

10. Kilian M, Chapple IL, Hannig M, Marsh PD, Meuric V, Pedersen AM, Tonetti MS, Wade WG, Zaura E. The oral microbiome - an update for oral healthcare professionals. British dental journal 2016;221(10):657-666.

11. Koren O, Spor A, Felin J, Fak F, Stombaugh J, Tremaroli V, Behre CJ, Knight R, Fagerberg B, Ley RE, Backhed F. Human oral, gut, and plaque microbiota in patients with atherosclerosis. Proceedings of the National Academy of Sciences of the United States of America 2011;108 Suppl 1:4592-8.

12. Slocum C, Kramer C, Genco CA. Immune dysregulation mediated by the oral microbiome: potential link to chronic inflammation and atherosclerosis. Journal of internal medicine 2016;280(1):114-28.

13. Masi S, Orlandi M, Parkar M, Bhowruth D, Kingston I, O'Rourke C, Virdis A, Hingorani A, Hurel SJ, Donos N, D'Aiuto F, Deanfield J. Mitochondrial oxidative stress, endothelial function and metabolic control in patients with type II diabetes and periodontitis: A randomised controlled clinical trial. International journal of cardiology 2018;271:263-268. 
14. Masi S, Gkranias N, Li K, Salpea KD, Parkar M, Orlandi M, Suvan JE, Eng HL, Taddei S, Patel K, Darbar U, Donos N, Deanfield JE, Hurel S, Humphries SE, D'Aiuto F. Association between short leukocyte telomere length, endotoxemia, and severe periodontitis in people with diabetes: a cross-sectional survey. Diabetes care 2014;37(4):1140-7.

15. Masi S, Salpea KD, Li K, Parkar M, Nibali L, Donos N, Patel K, Taddei S, Deanfield JE, D'Aiuto F, Humphries SE. Oxidative stress, chronic inflammation, and telomere length in patients with periodontitis. Free radical biology \& medicine 2011;50(6):730-5.

16. Masi S, D'Aiuto F, Martin-Ruiz C, Kahn T, Wong A, Ghosh AK, Whincup P, Kuh D, Hughes A, von Zglinicki T, Hardy R, Deanfield JE, scientific N, data collection t. Rate of telomere shortening and cardiovascular damage: a longitudinal study in the 1946 British Birth Cohort. European heart journal 2014;35(46):3296-303. 


\section{Figure legend}

Figure 1. Potential mechanisms accounting for the increased risk of cardiovascular disease in patients with periodontitis and/or other oral inflammatory/infectious diseases. Bacteria causing oral infection can translocate into the systemic circulation activating inflammatory cells and vascular endothelial cells. They might also localise in the vascular wall, potentially promoting progression of atherosclerosis. Activated inflammatory cells undergo replication and produce free radicals (ROS) that might result in telomere shortening, activation of the DNA damage response and cellular dysfunction. Pro-inflammatory cytokines with immunomodulatory functions are produced by both endothelial cells and inflammatory cells, with further amplification of the systemic inflammatory response and increased systemic oxidative stress. Furthermore, activated endothelial cells express adhesion molecules that promote translocation of inflammatory cells and lipids in the subendothelial space, ultimately leading to the formation of the atherosclerotic plaque. 